\title{
Inkjet printing of insulin microneedles for transdermal delivery
}

\author{
Steven Ross, Nicolaos Scoutaris ${ }^{1}$, Dimitrios Lamprou ${ }^{2}$, David
} Mallinson ${ }^{2}$, Dennis Douroumis ${ }^{1}$

${ }^{1}$ Faculty of Engineering and Science, University of Greenwich, Medway Campus, Chatham Maritime, Kent ME4 4TB, UK

${ }^{2}$ Strathclyde Institute of Pharmacy and Biomedical Sciences, University of Strathclyde, The John Arbuthnott Building, Glasgow G40NR, Scotland

\footnotetext{
${ }^{1}$ Corresponding author, Faculty of Engineering and Science, University of Greenwich, Medway Campus, Chatham Maritime, Kent ME4 4TB, UK. Tel.: +44 (0) 208331 8440; Fax: +44 (0) 208331 9805. E-mail address: D.Douroumis@gre.ac.uk (D. Douroumis).
} 


\begin{abstract}
Inkjet printing technology was used to apply insulin polymeric layers on metal microneedles for transdermal delivery. A range of various polymers such as gelatin (GLN), polyvinyl caprolactame-polyvinyl acetate-polyethylene glycol (SOL), poly(2-ethyl-2-oxazoline) (POX) and trehalose (THL) were assessed for their capacity to form thin uniform and homogeneous layers that preserve insulin intact. Atomic force microscopy (AFM) showed homogeneous insulin - polymer layers without any phase separation while SOL demonstrated the best performance. Circular discroism (CD) analysis of rehydrated films showed that insulin's alpha helices and $B-$-sheet were well preserved for THL and SOL. In contrast, GLN and POX insulin layers revealed small band shifts indicating possible conformational changes. Insulin release in Franz diffusion cells from MNs inserted into porcine skin showed rapid release rates for POX and GLN within the first $20 \mathrm{~min}$. Inkjet printing was proved an effective approach for transdermal delivery of insulin in solid state.
\end{abstract}

\title{
1. Introduction
}

Insulin medication is regarded as the most effective treatment for type 1 diabetes, with all type 1 and some type 2 diabetics requiring insulin injections to achieve glycaemic control (Hemmingsen et al, 2011). Insulin is typically given to the patient through self-administered subcutaneous needle injection. However, studies have found that these methods are inconvenient and painful, which has led to a decrease in compliance (Asche et al, 2010). It has been found that 1 out of 4 insulin dependent diabetic patients experience anxiety regarding self-injection. This contributed to the more worrying statistic that nearly $65 \%$ of type 1 and 2 diabetic patients are not confident in their ability to manage their disease by themselves (Korytkowski et al, 2005). This statistic is further emphasized by the fact that approximately $20 \%$ of children place their injections in and around the same area (Aronson, 2012). Repeated injections around the same spot has been strongly associated with skin thickening around the injection sites. Studies have shown a correlation between increased skin thickening and poor glycaemic control, suggesting that the increased thickness around injection sites prevent the correct dose of insulin from being administered (Derraik et al, 2014).

Due to the drawbacks of hypodermic injection of insulin and lack of suitable alternatives, microneedles are drawing attention as an alternative pathway for insulin delivery. Microneedles $(\mathrm{MN})$ are narrow needles of the order of microns with lengths up to $1 \mathrm{~mm}$ which can pierce through the stratum corneum, but will not penetrate deep enough into the skin to stimulate nerve receptors, making the procedure painless. MNs have already proven an attractive medical devices for transdermal delivery for a wide range of active substances such as vaccines, proteins, anticancer drugs, oligonucleotides and DNA (Arora et al., 2008, Kim et al., 2014). It has been found that MN insertion pain was significantly lowered when compared to injection via subcutaneous injection.

A hindrance in the transdermal delivery of therapeutic proteins is that they are labile under unfavourable conditions. Insulin can become denatured due to hydrolytic reaction or possibly transformed to components of a higher molecular weight, when delivered through 
transdermal mechanisms (Vimalavathini et al, 2009. Ye et al., 2008). Therefore, to avoid issues with insulin stability, surfactants must be added to the formulation, to stabilize the structure of the product (Fonte et al., 2012). However, this can present another obstacle as the substance added could potentially increase the dissolution time of the formulation, meaning insulin is absorbed into systemic circulation at a slower rate.

This study sought to develop an insulin-polymer solution which can allow the greatest uptake of drug onto the microneedle array and rapidly deliver the highest amount of drug into the skin. Previous studies have used polymers as insulin stabilizers, due to their quick dissolution time within the skin (Chen et al, 2012. Ling et al, 2013). Gelatin is as a potential insulin stabilizer due to its biodegradability, biocompatibility, non-immunogenicity and filmforming ability (Thyagarajapuram et al, 2007). This is ideal for insulin delivery via microneedle as it should not interfere with the therapeutic action of the insulin, instigate no immune reaction and the film forming properties should allow for easy uptake onto the microneedle. Gelatin has been shown to promote transmucosal delivery of insulin by increasing the permeability of the drug through epithelial membranes (Seki et al., 2005). This evidence justifies the choice of gelatin as an insulin stabilizer.

Trehalose is established as an excellent protein stabilizer and past studies have shown to be uniquely suitable for stabilizing proteins due to its low chemical reactivity, non-reducing nature and a high affinity for water molecules. (Kaushik et al, 2003.). However, studies using mouse models have found that Trehalose suppresses adipocyte hypertrophy and mitigates insulin resistance. This could be cause for concern, as a resistance of the delivered insulin would undermine the stabilizing properties of the polymer (Arai et al, 2013). Another potential stabilizer analysed is polyvinyl caprolactam-polyvinyl acetate-polyethylene glycol graft copolymer (Soluplus®). Various studies have shown Soluplus® to promote fast drug release and be used as a solubility enhancer (Homayouni et al., 2014). Soluplus ${ }^{\circledR}$ has previously been used to increase the bioavailability through oral drug delivery and has been shown to adequately stabilize proteins (Paaver et al., 2014).

Another potential insulin stabilizer is Poly(2-oxazoline) (POx) where numerous studies have shown POx to be a highly efficient drug delivery platform which offers low toxicity, low complement activation and has extremely high loading capacities for hydrophobic drugs; all of which benefit insulin delivery as a large amount can then be delivered safely without becoming denatured (Luxenhofer et al., 2010. Tong et al., 2011). POxylated proteins have been shown to better enhance the transport and intracellular delivery of proteins than the more conventional method of PEGulated proteins (Luxenhofer et al., 2012).

The microneedles were coated via inkjet printing as the method has been shown to deliver highly uniform, reproducible and accurate coatings at various drug-polymer ratios (Uddin, et al, 2015)

\section{Materials Methods}

\subsection{Materials}

Insulin, from bovine pancreas (Sigma-Aldrich, St Louis, MO) as higher MW model macromolecule. Gelatin, (GLN) from porcine skin, Type A, 90-110 Bloom (Sigma-Aldrich, St Louis, MO) as stabiliser. Trehalose dehydrate (THL), from Saccharomyces cerevisiae (Sigma-Aldrich, St Louis, MO), as stabiliser. Soluplus ${ }^{\circledR}$ (SOL) a co-polymer of polyvinyl 
caprolactame-polyvinyl acetate-polyethylene glycol, (BASF, Minden), as stabilizer. Poly(2ethyl-2-oxazoline) (POX) as stabilizer. Phosphate buffer saline (PBS) $0.153 \mathrm{M}$, prepared using: Na2HPO4.12H2O $2.86 \mathrm{~g} / \mathrm{l}(8 \mathrm{mM}), \mathrm{KH} 2 \mathrm{PO} 40.2 \mathrm{~g} / \mathrm{l}(1.5 \mathrm{mM}), \mathrm{NaCl} 8.0 \mathrm{~g} / \mathrm{l}$ and $\mathrm{KCl}$ $0.19 \mathrm{~g} / \mathrm{l}(2.5 \mathrm{mM})$.

\subsection{Microneedle Fabrication}

Arrays of solid microneedles) were fabricated by cutting needle structures from stainless steel sheets (SS 304, $75 \mathrm{~mm}$ thick; McMaster-Carr, Atlanta, GA, USA) using an infrared laser (Reso- netics Maestro, Nashua, NH, USA). The fabricated arrays consisted of 50 needles per $\mathrm{cm}^{2}$ and a needle tip radius of $10 \mu \mathrm{m}$. Initially, the shape and orientation of the arrays were drafted in a CAD file (AutoCAD; Autodesk, Cupertino, CA, USA), by using the laser-control software. The laser beam traced the desired shape of the needle, which ablated the metal sheet and created the needles in the plane of the sheet. The laser was operated at $1000 \mathrm{~Hz}$ at an energy density of $20 \mathrm{~J} / \mathrm{cm} 2$ and required approximately 4 min to cut an array. The metal sheet with needles on it was cleaned in hot water (Alconox, White Plains, NY, USA) and rinsed with DI water. Each needle was then manually bent at $90^{\circ}$ out of the plane of the sheet. The needles were electropolished in a bath containing a 6:3:1 mixture by volume of glycerin, phosphoric acid, and water (Fisher Scientific, Atlanta, GA, USA) to remove debris. This electropolishing process reduced the needle thickness to $50 \mu \mathrm{m}$.

\subsection{Inkjet printing process for MN coating}

Inkjet printing was performed with a Nanoploter II (Gesim, Germany) where the MNs are mounted on a specifically designed holder and positioned at $45^{\circ}$ relative to the dispenser (Fig. 1). The drug/polymer solutions were jetted through a piezodriven dispenser (PicPip 300) onto

the $\mathrm{MN}$ surface in the form of fine droplets of approximately $300 \mathrm{pl}$. The appropriate drug/polymer amounts were coated through various coating cycles. For each coating cycle, 6 dots of a coating formulation every $50 \mu \mathrm{m}$ were dispensed longitudinally to the axis of each $\mathrm{MN}$ and the process was repeated for 50 jetting cycles to coat the desired amounts. The dispenser is a piezoelectric ceramic that is deformed upon voltage appliance, thus ejecting a droplet from the nozzle at a speed 1-5 m/s ("drop on demand"). Due to the MN design a simple coating algorithm that targets the needle edge was designed. In this algorithm the numbers of spots, the spots/needle and the distance per spot can be determined. The operation process is controlled by a stroboscope, which controls if the dispenser jets according to the desired standard droplet size. Before the coating process starts, the dispenser deposits one droplet onto a water sensitive paper producing a dark spot in a yellow background (Fig 1a). This is used to determine the exact position of the dispenser. Specifically, an algorithm for low - contrast images detects the spot on the paper and calculated its XY deviation from the theoretical position (Fig 1b). Stroboscopic image capture provides real-time analysis of pipette performance both before and after sample dispensing. Fig. 1c shows the dispenser passing in front of the stroboscope. After the accomplishment of each cycle the dispenser passed in front of the oscilloscope to check whether the dispensing was according to the standards. The droplet diameter allows estimation of the dispensed volume calculated through image analysis. If, for any reason, a sample is not dispensed as instructed (e.g. empty sample 
well), the software logs this and allows for repetition of respective sample printing, thus ensuring completion of the task.

\subsection{Coating formulations}

Polymers were dissolved in deionized water prior to the addition of the insulin solution. Table 1 below shows the different insulin-polymer ratios. For the estimation of insulin-coated amounts for each formulations three MN arrays were fully dissolved separately in $20 \mathrm{ml}$ of phosphate buffer ( $\mathrm{pH}$ 7.4) and the insulin was determined by HPLC analysis.

\subsection{Scanning electron Microscopy (SEM)}

The microneedle arrays were mounted on an aluminium stub by the use of doubled-sided carbon adhesive tape. The microneedles were analysed using scanning electron microscope (JEOL JSM-5310V, Japan). All un-coated and coated MNs was characterised at low vacuum (LV mode) and accelerating voltage $(20 \mathrm{kV})$ using tilted angle of $83^{\circ}$. All images were captured digitally during analysis.

\subsection{Atomic force Microscopy (AFM)}

AFM analysis was performed on tapping mode using an easyscan AFM (Nanosurf, Lielestal, Switzerland). Tap 190Al-G cantilevers were used (Budgetsensor, Sofia, Bulgaria).The drive amplitude and the relative set point were chosen in a way that the intermittent force between the oscillated tip and the substrate would be minimum and the scan areas was $5 \mu \mathrm{m}$. The analysis of the images was performed using the nanoscan software.

As it was difficult to perform AFM analysis on the microneedle without damaging it, the solutions were deposited onto the microneedles substrate by using the nano-plotter and placing them on 45 degrees. The number of layers were the same as for the other studies.

\subsection{Circular Dichroism (CD)}

Insulin - polymer films were suspended in $200 \mu \mathrm{L} \mathrm{PBS} \mathrm{(pH} \mathrm{7.4)} \mathrm{and} \mathrm{the} \mathrm{far-UV} \mathrm{spectra} \mathrm{were}$ recorded between 200 and $280 \mathrm{~nm}$ on a Chirascan CD spectrometer (Applied Photophysics, UK) with the lamp supplied with a flow of nitrogen. $50 \mu \mathrm{L}$ of the solution were added to a $0.1 \mathrm{~mm}$ path-length quartz cuvette (Hellma, UK) and the measurements were carried out at $20{ }^{\circ} \mathrm{C}$ ( $1 \mathrm{~nm}$ bandwidth resolution and $1 \mathrm{~s}$ acquisition time). Typically, at least two scans were recorded, and baseline and PBS spectra were subtracted from each spectrum. Data were processed using Applied Photophysics Chirascan Viewer and Microsoft Excel. The protein and polymer concentration spectra were normalised by scaling relative to the magnitude of the negative maximum (similar approach was used by Schmidt who normalised the value at $209 \mathrm{~nm})$ (Schmidt et al, 2014).

\subsection{Skin preparation for in-vitro MN testing}

Filter paper was soaked in water and placed in petri dish. A small amount of phosphate buffer at $7.4 \mathrm{pH}$ was added. This will prevent a change in the skin cells $\mathrm{pH}$, maintaining it at the same level as in the body. The abdominal area of porcine skin is very fatty, which can prevent penetration of microneedles. Fatty areas were removed with scalpel. Porcine skin was 
pinned to polystyrene block and wiped with $70 \%$ ethanol. The skin was then cut at $750 \mu \mathrm{m}$ by applying the dermatome at an angle of $\pm 45^{\circ}$. The skin was cut at $750 \mu \mathrm{m}$ as the microneedles measured 600 in length. This will allow the microneedles to penetrate the skins outer layers and deliver the drug directly into the dermis. The thickness of skin was measured by using a micrometer after placing a cover slip as a support base. The thickness of the cover slip was measured alone followed by the skin and cover slip together; the actual thickness was determined by subtracting one measurement from the other. Tissue disks of the required dimensions were cut for the Franz diffusion cells using a blade. The skin tissue (750-900 mm thick) was placed in PBS ( $\mathrm{pH}$ 7.4) for $2 \mathrm{~h}$. Padgett Dermatome and dermatome blades were purchased from Integra LifeTM Sciences Corporation (PA, USA).

\subsection{In-vitro permeability studies by using Franz diffusion cells}

The phosphate buffer (PBS) was maintained at $\mathrm{pH} 7.4$ and filled into the Franz diffusion cells (Permegear Inc.). A fraction collector (Gilson FC 204, Middleton, USA) was attached the water bath (Thermofisher scientific, Newington, USA) and PBS was supplied through a pump (Minipuls 2, Gilson, Luton, UK). Each cells were calibrate precisely with PBS at a rate 6-6.5 ml per hour. The receptor arm was sealed with plastic cap and the donor compartments were sealed with a cover slip to avoid sample evaporations. The constant temperature of the experiment was maintained at $37^{\circ} \mathrm{C}$ and an applied skin surface temperature of $32^{\circ} \mathrm{C}$. The cells were equilibrated up to 30 minutes before introducing test formulations to the donor compartments. The integrity of epidermal sheets was monitored for a while prior to start each experimental method.

The drug coated MN arrays were applied for 30 s onto the skin with an applied force of $7 \mathrm{~N}$ using the TA HD plus texture analyser (Stable micro system, Surrey, UK). Dislodge-ment of the MN arrays was prevented using an adhesive tape (3M, UK). Subsequently, the skin samples with the inserted MN arrays were mounted in the Franz diffusion cells with the stratum corneum on the topside. The drug cumulative release was measured for each formulation in triplicates over a $2 \mathrm{hr}$ period with a sample being taken every 10 minutes for the first hour and every 30 minutes for the second hour.

\subsection{HPLC analysis}

The amount of insulin present in the receptor fluid was determined using HPLC analysis. An Agilent technologies 1200 series HPLC system (AgilentTechnologies, Cheshire, UK) equipped with a Phenomenex Jupiter $5 \mathrm{u}$ c18 $300 \AA$, LC Column ( 250 x 4.60mm, particle size $5 \mu \mathrm{m}$, Macclesfield, UK) was used. The mobile phase consisted of water with $0.1 \%$ TFA and acetonitrile with $0.1 \%$ TFA $(65: 35 \mathrm{v} / \mathrm{v})$, with a $1 \mathrm{ml} / \mathrm{min}^{-1}$ flow rate (Liu et al., 2012). The eluent was monitored with a UV detector at $214 \mathrm{~nm}$, the column was equilibrated to $35^{\circ} \mathrm{C}$ and the injection volume was $20 \mu \mathrm{m}$. The results were integrated using Chemstation ${ }^{\circledR}$ software. The drug amount coated on the microneedle was quantified by placing the microneedle array in $10 \mathrm{ml}$ of deionised water and sonicating for 10 minutes to dissolve the coating layer. The insulin amount present was determined by HPLC analysis.

\section{Results and Discussion}

\subsection{Jet coating}


In general the quality of the coating depends on the size of the dispenser, the voltage applied and the duration of the pulse. Our evaluation showed that tips with $300 \mathrm{pl}$ aqueous droplet volume are suitable for microneedles coating. Tips generating smaller volumes (100 pl) can result in tip clogging. Moreover, a smaller tip cannot dispense such concentrated solutions as it requires less viscous solutions. In terms the voltage applied, it has been proved that the fluid volume ejected from the dispenser and as a consequence the droplet size is a linear function of the voltage (Tekin et al., 2008). An increase in the applied voltage for a given coating solution will lead to an increase in the diameter of the droplets. However, an increase of pulse duration will result in more complicated and periodic behaviour (Tsai et al, 2008). Also, increasing the voltage, the ejection velocity of the drop will increase and hence the impact of the drop onto the needle surface will be bigger causing splashing. Therefore, it was found that the optimal applied voltage and pulse duration during the coating process were $100 \mathrm{~V}$ and $60 \mu \mathrm{sec}$ respectively. The droplets had a consistent particle size of 100 $120 \mu \mathrm{m}$ without any satellites as observed from the stroboscope.

As previously mentioned (Uddin et al.) the distance of the nozzle from the microneedle is of the utmost importance. Due to the small generated droplets the dispenser must be as close as possible to the surface of microneedles. Therefore, the microneedles were positioned at $45^{\circ}$ relative to the dispenser. During the process the height of the dispenser changes according to the equation below in order to keep the distance between the dispenser and the microneedle at a constant value.

$\Delta \mathrm{L}=\mathrm{a} * \sin 45^{\circ}$

Where $\Delta \mathrm{L}$ is the height increase of the nozzle from the microneedle after dispensing of each droplet and $\mathrm{a}$ is the distance of each spot (droplet).

Fig. 2 illustrates a typical coating of insulin with THL, which is the result of 45 cycles. As it is clear that the deposited layers are spotted on the microneedle surface without any coating on the substrate or droplet satellites. By rotating the MN array it is possible to coat both sides of each needle and deposit equal amounts of coating material. Each microneedle was coated accurately with $110 \pm 10 \mu \mathrm{g}$ of insulin in order to avoid the deposition of bulky layers on the needle surface. As shown in Fig.2 the applied coatings were uniform and reproducible without any defects. On of the main advantages of inkjet printing compared to conventional coating techniques is the negligible material losses, high accuracy, reproducibility and the easiness of process scale - up. Ink jetting can be also used to apply aqueous or organic solvent coatings in routine manner. Inkjet printing results in a very small size of the dispensed droplets and the formation of film layers is rapid due to the fast evaporation of the coating medium.

\section{$3.2-A F M$}

AFM has been previously used to investigate the stiffness/roughness of polymeric films and the homogeneity/uniformity as well (Salerno et al., 2010). Fig. 3 illustrates the topographic image of pure polymers. The studies showed that inkjet printing produced smooth polymeric surfaces with SOL demonstrating the best performance (Table 2). When insulin was incorporated in the polymeric solutions at very high loadings $(50: 50 \mathrm{wt} / \mathrm{wt})$ the printed layers became rougher, with the exception of the insulin - POX layers. However, even in this occasion the estimated values of the film roughness remained small enough 
demonstrating the formation of smooth layers. In general, the roughness of the coating should be as smooth as possible to avoid the removal and consequently deposition of the coating layers on the skin during the MN piercing.

Moreover, all of the inkjet printed insulin - polymer formulations presented homogenous and uniform layers without any phase separation observed. As previously shown the homogeneity of the applied inkjet dispensed layers is a prerequisite to achieve fast release of insulin as previous studies shown (Uddin at al. 2015). The AFM analysis was in a good agreement with the smooth layers observed in the SEM images.

\section{$3.3 C D$}

In order to evaluate the effect of inkjet dispensing and the formation of thin polymer layers where insulin is present at solid state, CD analysis was conducted as shown in Table 3. $\mathrm{CD}$ is a well known spectroscopic technique that provides valuable information related to denaturation and helix coil transformations of insulin. In these studies all MN coated formulations were kept at $4 \mathrm{oC}$ prior CD evaluation in order to eliminate any possible insulin degradation. As shown in Fig. 5 insulin absorption bands associated with alpha helices and $\beta-$ sheet are seen at $208 \mathrm{~nm}$ and $222 \mathrm{~nm}$ respectively (Gopal et al, 2012). The obtained spectra are in agreement with previously reported studies especially those representing the alphahelical structure of insulin (Patil et al, 2014). These studies demonstrated that the relative ellipticity of the $222 \mathrm{~nm}$ band with respect to the $208 \mathrm{~nm}$ band was between 70 and $90 \%$. As shown in Fig. 6 the insulin/THL and insulin/SOL coatings showed almost identical bands with those of the insulin sample (i.e. RI209 = RI222) suggesting very good physical stability of insulin within the polymer layers. For the insulin/GLN coatings a small minimal shift from $222 \mathrm{~nm}$ spectra was observed. This type of shift was identified by Zhang et al., (2010) for insulin encapsulated in nanoparticles and are attributed to interference of GLN with insulin without however affecting significantly the a-helix/ $/$ - sheet of the protein.

In contrast a significant change can be seen for the insulin/POx coatings at the second negative maximum at 206, which has completely disappeared. This type of behaviour could be related to manipulation or processing conditions, but also on strong molecular interactions between the insulin and the polymer (Zhang et al, 2010). This type of interactions can effectively lead to some conformational changes, thus exposing hydrophobic areas to outside, resulting in reduced solubility and an increased tendency of aggregation. This can also result in dissociation of insulin hexamers into monomers and dimers. CD analysis of insulin/POx coatings indicates that insulin undergoes one of the aforementioned interactions, which requires further investigation.

\subsection{Release studies}

To identify which drug stabilizer offers the greatest and most rapid delivery, the amount of insulin release from the coated MNs was estimated by using Franz cell diffusion. Using Franz cell diffusion a permeant will diffuse from a vehicle in the donor chamber, through an artificial or biological membrane into receptor fluid, which will drip into a receptor chamber. In this case as the polymer stabilizer dissolves, the insulin will diffuse from the microneedle through the porcine skin into the continuously flowing receptor fluid. 
From the results shown in Fig. 7, it is clear that SOL allowed for the most rapid release of insulin, with $65 \%$ being released within the first 10 min while $95 \%$ under 30 min.. GLN showed a similar release profile indicating that both GLN and SOL dissolve quickly enough for insulin to rapidly delivered into the bloodstream. However POX and THL have a poor first release showing that both coating formulations are poorly suited to deliver insulin into circulation. POX had an especially poor release delivering with only $42 \%$ of insulin after 30 min, compared to GLN and SOL.

The long-term usability of microneedles as a form on insulin delivery hinges on the convenience of their use and dosage control. Through hypodermic injection, the complete dose is delivered within seconds and the insulin may rapidly take effect. Therefore for microneedles to become the preferred choice, insulin must be released as quickly as possible. Current data suggests that the optimal injection-to-meal (IMI) interval for both type 1 diabetics is between 20-30 minutes (Müller et al, 2011). Therefore, based on this data it is important for the polymer stabilizer to allow the insulin to be absorbed within this time frame. The microneedles loaded with the SOL and GLN solutions released over $80 \%$ of loaded insulin under $30 \mathrm{~min}$, suggesting that both could serve as suitable insulin carriers. However, emerging research has downplayed the importance of IMI in type 2 diabetics (Müller et al, 2013). For a diabetic patient to achieve optimal blood glucose control, the amount of insulin injected must correlate with food intake after injection. Therefore, it is important that the entire injected amount of insulin be absorbed into circulation; if even a small amount of insulin remains undelivered, it could have a potentially great effect on blood-glucose levels ( $\mathrm{Ma}$ et al, 2006). After $40 \mathrm{~min}$ the microneedle coated with SOLinsulin released all of the active substance and only a small amount being released outside of the optimal IMI. From this it can be concluded that SOL is the best and only real success of the experiment, releasing its full amount within an acceptable timeframe.

Despite its fast initial release, GLN did not release its full amount of loaded insulin. It was however only a minor amount, which remained, undelivered and could be attributed to the fact that GLN could potentially create a viscous solution in the MN proximity. This would eventually slowdown the release rates of insulin. Future rheological studies for different GLN grades and also insulin/GLN ratios would lead to further optimization. The CD studies revealed a small shift from $222 \mathrm{~nm}$ in the insulin/GLN coatings. This could potentially cause a disassociation of insulin hexamers. An investigation into how strong these interactions are would reveal the full effect gelatin exerts on the insulin molecule.

The worst performing polymer stabilizer was POX, with a slow release of less than half of the loaded insulin. This can be attributed to the conformational changes observed in the CD studies. The strong reactions between POX and insulin could have potentially caused changes in structure which possibly lead to decreased solubility. Therefore, the POX-Insulin complex would not readily dissolve once injected into the skin, meaning a large portion of the insulin loaded would not be absorbed into circulation. The slow release of POX can also be attributed to the breakdown of insulin hexamers into monomers and dimers will cause the insulin molecules to exhibit limited bioavailability after administration (Patil et al, 2014).

Trehalose also performed poorly in this experiment, with a slow release time and a failure to deliver all of its loaded insulin. This result is surprising given THL proven capability as an effective protectant (Rodrigues et al, 2008). Further studies into the hydration rates of the polymer could help explain this result. 


\section{Conclusions}

Inkjet printing was employed to develop insulin polymeric layers on metal microneedles for transdermal layers. Surface characterization of the printed layers showed homogeneous and uniform layers without any defects on the metal surface. Circular dichroism revealed stable insulin layers with intact alpha helices and $\beta$-sheets for THL and SOL while possible structural configurations were observed for GLN and THL. Most importantly rapid insulin release rates were observed fro SOL and GLN within the first 20 min suggesting that solidstate insulin delivery via microneedles is feasible.

\section{Conflict of Interest}

The authors declare that they have no conflict of interest

\section{References}

Arai, C., Miyake, M., Matsumoto, Y., Mizote, A., Yoshizane, C., Hanaya, Y., Koide, K., Yamada, M., Hanaya, T., Arai, S. and others. Trehalose prevents adipocyte hypertrophy and mitigates insulin resistance in mice with established obesity. Journal of nutritional science and vitaminology, 2013. 59(5), pp.393--401.

Aronson, R. The Role of Comfort and Discomfort in Insulin Therapy. Diabetes Technology \& Therapeutics. 2012. 14(8), pp.741-747.

Arora, A., Prausnitz, M. and Mitragotri, S. Micro-scale devices for transdermal drug delivery. International Journal of Pharmaceutics. 2008. 364(2), pp.227-236.

Asche, C., Shane-McWhorter, L. and Raparla, S. Health Economics and Compliance of Vials/Syringes Versus Pen Devices: A Review of the Evidence. Diabetes Technology \& Therapeutics. 2010. 12(S1), pp.S-101-S-108.

Chen, M., Ling, M., Lai, K. and Pramudityo, E. Chitosan. Microneedle Patches for Sustained Transdermal Delivery of Macromolecules. Biomacromolecules, 2012. 13(12), pp. $4022-31$

Derraik, J., Rademaker, M., Cutfield, W., Peart, J., Jefferies, C. and Hofman, P. Poorer glycaemic control is associated with increased skin thickness at injection sites in children with type 1 diabetes. Int J Pediatr Endocrinol. 2014. 2014(1), p.2.

Fonte, P., Soares, S., Costa, A., Andrade, J., Seabra, V., Reis, S. and Sarmento, B. Effect of cryoprotectants on the porosity and stability of insulin-loaded PLGA nanoparticles 
after freeze-drying. Biomatter. 2012. 2(4), pp.329-339.

Gopal R, Park J, Seo C, Park Y. Applications of Circular Dichroism for Structural Analysis of Gelatin and Antimicrobial Peptides. IJMS. 2012;13(12):3229-3244.

Hemmingsen, B., Lund, S., Gluud, C., Vaag, A., Almdal, T., Hemmingsen, C. and Wetterslev, J. Intensive glycaemic control for patients with type 2 diabetes: systematic review with meta-analysis and trial sequential analysis of randomised clinical trials. $B M J$. 2011. 343:d6898

Homayouni, A., Sadeghi, F., Varshosaz, J., Afrasiabi Garekani, H. and Nokhodchi, A. Promising dissolution enhancement effect of soluplus on crystallized celecoxib obtained through antisolvent precipitation and high pressure homogenization techniques. Colloids and Surfaces B: Biointerfaces. 2014. 122, pp.591-600.

Kaushik, J. and Bhat, R. Why Is Trehalose an Exceptional Protein Stabilizer: An analysis of the thermal stability of protines in the presence of the compatible osmolyte Trehalose Journal of Biological Chemistry. 2003. 278(29), pp.26458-26465.

Kim, N., Lee, M., Kim, K., Lee, J., Lee, K., Park, J., Matsumoto, Y., Jo, D., Lee, H., Lee, D. and Jeong, J. Polyplex-releasing microneedles for enhanced cutaneous delivery of DNA vaccine. Journal of Controlled Release, 2014. 179, pp.11-17.

Korytkowski, M., Niskanen, L. and Asakura, T. FlexPen®: Addressing issues of confidence and convenience in insulin delivery. Clinical Therapeutics. 2005. 27, pp.S89S100.

Ling, M. and Chen, M. Dissolving polymer microneedle patches for rapid and efficient transdermal delivery of insulin to diabetic rats. Acta Biomaterialia. 2013. 9(11), pp.89528961.

Liu, S., Jin, M.N., Quan, Y.S., Kamiyama, F., Katsumi, H., Sakane, T., Yamamoto A. The development and characteristics of novel microneedle arrays fabricated from hyaluronic acid, and their application in the transdermal delivery of insulin. J. Control. Rel. 2012. 161, 933-941

Luxenhofer, R., Han, Y., Schulz, A., Tong, J., He, Z., Kabanov, A. and Jordan, R. Poly(2oxazoline)s as Polymer Therapeutics. Macromol. Rapid Commun. 2012. 33(19), pp.16131631.

Luxenhofer, R., Schulz, A., Roques, C., Li, S., Bronich, T., Batrakova, E., Jordan, R. and 
Kabanov, A. Doubly amphiphilic poly(2-oxazoline)s as high-capacity delivery systems for hydrophobic drugs. Biomaterials, 2010. 31(18), pp.4972-4979.

Ma, R. and Tang, T. Current Strategies to Improve the Bioactivity of PEEK. International journal of molecular sciences, 2014. 15(4), pp.5426--5445.

Maderuelo, C., Zarzuelo, A. and Lanao, J. (2011). Critical factors in the release of drugs from sustained release hydrophilic matrices. Journal of Controlled Release, 154(1), pp.219.

Müller N, Frank T, Kloos C, Lehmann T, Wolf G, Muller U. Randomized Crossover Study to Examine the Necessity of an Injection-to-Meal Interval in Patients With Type 2 Diabetes and Human Insulin. Diabetes Care. 2013;36(7):1865-1869.

Müller N, Frank T, Kloos C, Lehmann T, Wolf G, Muller U. Randomized Crossover Study to Examine the Necessity of an Injection-to-Meal Interval in Patients With Type 2 Diabetes and Human Insulin. Diabetes Care. 2013;36(7):1865-1869.

Paaver, U., Tamm, I., Laidmäe, I., Lust, A., Kirsimäe, K., Veski, P., Kogermann, K. and Heinämäki, J. Soluplus Graft Copolymer: Potential Novel Carrier Polymer in Electrospinning of Nanofibrous Drug Delivery Systems for Wound Therapy. BioMed Research International. 2014. 2014, pp.1-7.

Patil N, Devarajan P. Enhanced insulin absorption from sublingual microemulsions: effect of permeation enhancers. Drug Delivery and Translational Research. 2014;4(56):429-438.

Prausnitz, M. and Langer, R. Transdermal drug delivery. Nature biotechnology, 2008. 26(11), pp.1261--1268.

Rodrigues J, Paraguassú-Braga F, Carvalho L, Abdelhay E, Bouzas L, Porto L. Evaluation of trehalose and sucrose as cryoprotectants for hematopoietic stem cells of umbilical cord blood. Cryobiology. 2008; 56(2):144-151.

Salerno M, Dante S, Patra N, Diaspro A. AFM measurement of the stiffness of layers of agarose gel patterned with polylysine. Microsc Res Tech. 2010. 73(10), pp 982-90

Schmidt S, Uhlig K, Duschl C, Volodkin D. Stability and cell uptake of calcium carbonate templated insulin microparticles. Acta Biomaterialia. 2014;10(3):1423-1430.

Seki, T., Kanbayashi, H., Nagao, T., Chono, S., Tomita, M., Hayashi, M., Tabata, Y. and 
Morimoto, K. Effect of Aminated Gelatin on the Nasal Absorption of Insulin in Rats. Biological \& Pharmaceutical Bulletin. 2005. 28(3), pp.510-514.

Tekin, E., Smith, P. and Schubert, U. Inkjet printing as a deposition and patterning tool for polymers and inorganic particles. Soft Matter, 2008. 4(4), p.703.

Thyagarajapuram, N., Olsen, D. and Middaugh, C. Stabilization of proteins by recombinant human gelatins. Journal of Pharmaceutical Sciences. 2005. 96(12), pp.33043315.

Tong, J., Zimmerman, M., Li, S., Yi, X., Luxenhofer, R., Jordan, R. and Kabanov, A. Neuronal uptake and intracellular superoxide scavenging of a fullerene (C60)-poly(2oxazoline)s nanoformulation. Biomaterials, 2011. 32(14), pp.3654-3665.

Tsai, M. and Hwang, W. Effects of Pulse Voltage on the Droplet Formation of Alcohol and Ethylene Glycol in a Piezoelectric Inkjet Printing Process with Bipolar Pulse. MATERIALS TRANSACTIONS, 2008. 49(2), pp.331-338.

Uddin, M., Scoutaris, N., Klepetsanis, P., Chowdhry, B., Prausnitz, M. and Douroumis, D. Inkjet printing of transdermal microneedles for the delivery of anticancer agents. International Journal of Pharmaceutics. 2015. S0378-5173.

Vimalavathini, r. and Gitanjali, B. Effect of temperature on the potency \& pharmacological action of insulin. Indian Journal Of Medical Research. 2009. 130(2), pp.166-9.

Ye, H., Hill, J., Kauffman, J., Gryniewicz, C. and Han, X. Detection of protein modifications and counterfeit protein pharmaceuticals using isotope tags for relative and absolute quantification and matrix-assisted laser desorption/ionization tandem time-offlight mass spectrometry: Studies of insulins. Analytical Biochemistry. 2008. 379(2), pp.182-191.

Zhang, N., Li, J., Jiang, W., Ren, C., Li, J., Xin, J. and Li, K. Effective protection and controlled release of insulin by cationic $\hat{I}^{2}$-cyclodextrin polymers from alginate/chitosan nanoparticles. International Journal of Pharmaceutics. 2010. 393(1-2), pp.213-219. 
Table 1: Compositions of polymer and insulin solutions

\begin{tabular}{|c|c|}
\hline Formulations & $\begin{array}{c}\text { Compositions } \\
\text { (5mg/10ml) }\end{array}$ \\
\hline F1 & POX/Insulin \\
\hline F2 & GLN/Insulin \\
\hline F3 & THL/Insulin \\
\hline F4 & SOL/Insulin \\
\hline
\end{tabular}

Table 2: Surface roughness of pure polymers and polymer/insulin mixture after jetting with inkjet technique.

\begin{tabular}{|c|c|c|c|}
\hline Polymers & $\begin{array}{c}\text { Roughness } \\
(\mathbf{S a}) \mathbf{n m}\end{array}$ & Insulin Coatings & $\begin{array}{c}\text { Roughness } \\
(\mathbf{S a})(\mathbf{n m})\end{array}$ \\
\hline GLN & 1.70 & GLN & 2.29 \\
\hline POX & 3.40 & POX & 1.91 \\
\hline THL & 0.48 & THL & 3.13 \\
\hline SOL & 7.42 & SOL & 16.9 \\
\hline
\end{tabular}

Table 3 Values obtained from CD analysis for each experiment. Wavelengths of maxima and ellipticity relative to negative maxima.

\begin{tabular}{|c|c|c|c|c|c|}
\hline & Insulin/POX & Insulin/GLN & Insulin/THL & Insulin/SOL & $\begin{array}{c}\text { Insulin } \\
\text { (pure) }\end{array}$ \\
\hline $\begin{array}{c}\text { Negative } \\
\text { maximum, nm }\end{array}$ & 225 & 208 & 209 & 209 & 204 \\
\hline $\begin{array}{c}\mathbf{2}^{\text {nd }} \text { negative } \\
\text { maximum, nm } \\
\text { and relative } \\
\text { ellipticity }\end{array}$ & N/A & $225,53.6 \%$ & $223,80.8 \%$ & $223,68.8 \%$ & $210,98.9 \%$ \\
\hline
\end{tabular}




\begin{tabular}{|c|c|c|c|c|c|}
\hline $\begin{array}{c}\text { B-Sheet (222 } \\
\text { nm), relative } \\
\text { ellipticity }\end{array}$ & $95.4 \%$ & $52.2 \%$ & $77.4 \%$ & $69.2 \%$ & $100 \%$ \\
\hline $\begin{array}{c}\text { Alpha helix } \\
(\mathbf{2 0 8} \text { nm), } \\
\text { relative } \\
\text { ellipticity }\end{array}$ & $30.7 \%$ & $100 \%$ & $97.1 \%$ & $100 \%$ & $91.9 \%$ \\
\hline
\end{tabular}

\section{Figures Caption List}

Fig. 1 jet coating of microneedle

Fig. 1 a) Deposition of the droplet onto the water sensitive paper, b) positioning of the piezodispenser and the MN array for coating of insulin/polymer solutions, c) calculation of the XY deviation of the spot from the theoretical value and d) photographic image of the dispenser passing in front of the stroboscope during the coating.

Fig. 2: SEM image of a THL/insulin coated microneedle array.

Fig. 3: Topographic (left) and phase images of POX(a), GLN(b), THL(c) and SOL(d) produced by their deposition using inkjet dispensing

Fig. 4: Topographic (left) and phase imaging of dispensed mixtures of POX-insulin (a), GLN-insulin (b) THL-insulin (c) and SOL-insulin coating formulations by using inkjet dispensing

Fig. 5: CD spectra of insulin films rehydrated in PBS (pH 7.4).

Fig. 6: CD spectra of insulin films and GLN rehydrated in PBS (pH 7.4).

Fig. 7: in-vitro release profiles for insulin-polymer coating formulations using Franz diffusion cells $(\mathrm{n}=5)$. 